\title{
Zebrafish: A complete animal model to enumerate the nanoparticle toxicity
}

\author{
Chiranjib Chakraborty ${ }^{1 *+}$, Ashish Ranjan Sharma ${ }^{2+}$, Garima Sharma ${ }^{2}$ and Sang-Soo Lee ${ }^{2^{*}}$
}

\begin{abstract}
Presently, nanotechnology is a multi-trillion dollar business sector that covers a wide range of industries, such as medicine, electronics and chemistry. In the current era, the commercial transition of nanotechnology from research level to industrial level is stimulating the world's total economic growth. However, commercialization of nanoparticles might offer possible risks once they are liberated in the environment. In recent years, the use of zebrafish (Danio rerio) as an established animal model system for nanoparticle toxicity assay is growing exponentially. In the current in-depth review, we discuss the recent research approaches employing adult zebrafish and their embryos for nanoparticle toxicity assessment. Different types of parameters are being discussed here which are used to evaluate nanoparticle toxicity such as hatching achievement rate, developmental malformation of organs, damage in gill and skin, abnormal behavior (movement impairment), immunotoxicity, genotoxicity or gene expression, neurotoxicity, endocrine system disruption, reproduction toxicity and finally mortality. Furthermore, we have also highlighted the toxic effect of different nanoparticles such as silver nanoparticle, gold nanoparticle, and metal oxide nanoparticles $\left(\mathrm{TiO}_{2}, \mathrm{Al}_{2} \mathrm{O}_{3}, \mathrm{CuO}, \mathrm{NiO}\right.$ and $\left.\mathrm{ZnO}\right)$. At the end, future directions of zebrafish model and relevant assays to study nanoparticle toxicity have also been argued.
\end{abstract}

Keywords: Zebrafish, Nanoparticle, Toxicity, Animal model

\section{Background}

Now-a-days, nanotechnology encompasses an increasing impact on the industrial revolution accounting for multibillion-dollar business sector. Various industrial sectors, including tissue engineering, drug delivery, imaging, diagnostics, surface texturing, and bio-interfaces are currently using nanomaterials in their products [1,2]. Hence, with the growing business impact of nanotechnology, Business Communications Company (BCC) projected that the nanotechnology industry was approximately 7.6 billion USD market in 2013 which further has a potential to rise up to 1 trillion USD by 2020 [3]. Nanotechnology

\footnotetext{
*Correspondence: drchiranjib@yahoo.com; totalhip@hallym.ac.kr; 123sslee@gmail.com

${ }^{\dagger}$ Chiranjib Chakraborty and Ashish Ranjan Sharma contributed equally to this work

${ }^{1}$ Department of Bioinformatics, School of Computer and Information Sciences, Galgotias University, Greater Noida, Uttar Pradesh, India

${ }^{2}$ Institute of Skeletal Aging and Orthopedic Surgery, Hallym UniversityChuncheon Sacred Heart Hospital, Hallym University, Chuncheon, Gangwon-do 24252, Republic of Korea
}

is upcoming as a solution across a range of industrial problems and also acts as a crossroad for different enabling technologies like biotechnology, computational science, physical science, communications technology, cognitive science, and others $[4,5]$. Mihail Roco of the U.S. National Nanotechnology Institute visualized four generations of nanotechnology [6] and expected that the third generation is about to appear around 2010 with different types of nanosystems and thousands of their interacting components. In accordance, until 2013, 1814 nanoparticles products are commercially available in the market [7].

Nanotechnology is known for the designing, development, description, and applications of materials at nanometer scale. Furthermore, engineered nanodevices are finding an ever-expanding range of applications due to the possibility of versatile modifications in their shape, size, surface, and chemical properties. The surface of nanomaterials can be modulated according to their application such as for drug delivery the biocompatibility of the nanomaterials can be modified and their cell specific 
targeting ability can also be enhanced by attaching them with targeting ligand $[8,9]$. Presently, people are using a wide variety of commercially available nanoparticles for their daily utilities such as-(1) silver (Ag) nanoparticles are used in sheets and clothing, (2) titanium dioxide nanoparticles are used in different cosmetics, lotions and creams, (3) carbon nanoparticles are used in motorcycle and in bicycle, and (4) clay nanoparticles are used in beer bottles [10]. With the growing demand of nanoparticles and their commercial potential, the area of nanotoxicity has grown considerably during the last $10-15$ years and we are hoping that in near future it will address our serious concern. The area of nanotoxicity also addresses the regulatory aspects for the growing explosion in nanoparticle technology. The enhanced possibility of nanoparticle exposure and their toxic effects on consumers and environment is also a thoughtful issue to be highlighted [11].

In fact, there are an increasing number of literatures that documents the concern over toxicity for broad range of engineered nanoparticles/nanomaterials such as CNT, fullerenes, graphene metal nanoparticles, metal oxides nanoparticles, crystalline materials, amorphous materials and nano-sized polymers [12]. Various toxicity experimental assays/model organisms are used time to time for this purpose, such as in vitro cellular assays, multi-cellular model organisms (such as daphnia and sea urchin) [13] and higher animal models like mice [14]. With the use of the compound material from in vitro to in vivo experiments, it has been observed that the higher animal models are more valuable in comparison to simple experimental models. Each and every year, the number of engineered nanomaterials and their products are continuously increasing and there is a critical need to develop representative model organism, able to assess nanotoxicity accurately and to screen the nanoparticles at throughput level. In this regard, zebrafish as an in vivo model organism has attracted scientific interest because of its unique features (Fig. 1).

In this depth review, firstly we focused on the advantages of zebrafish that makes it a popular experimental animal model for various studies. Further, we described the different nano-toxicological assessment methods in detail. Afterwards, various nanomaterial toxicological studies conducted using zebrafish model have been emphasized. Finally, we also highlighted the drawbacks and future prospects of zebrafish model for nanotoxicity studies.

\section{Zebrafish: a popular experimental animal model}

The global acceptance of zebrafish as a modern experimental animal model is increasing gradually. This animal model is becoming popular in the fields of toxicology and biomedical research during both adult as well as

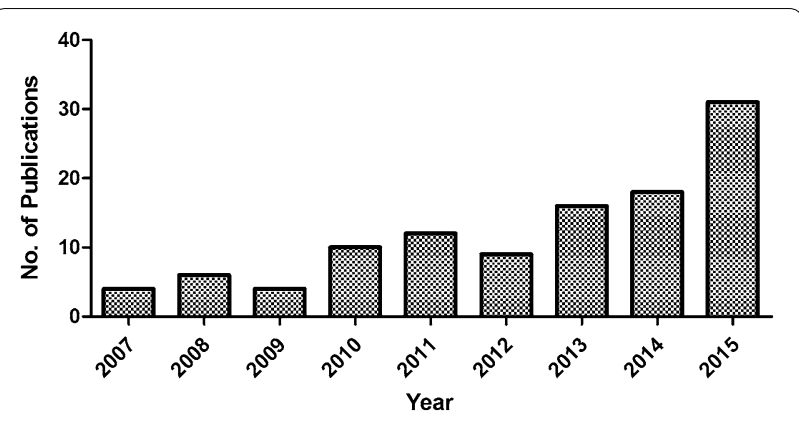

Fig. 1 Increasing trend in the publications in zebrafish research (2007-2015). Keyword ("nano" and "zebrafish") searched was performed from PUBMED, NCBI database. Search was conducted on 14th July 2016

embryonic stages. The reason for this wide recognition of zebrafishes as popular animal model is due to some exceptional set of characteristics it possesses. Some of them are their small size, very high reproducibility, quick development, transparency of the embryo and acquiescent to genetic as well as chemical screens $[15,16]$. Additionally, we can also find extensive literature on zebrafish experiments. It has been noted that zebrafish is a small sized animal and, therefore, it can be handled without any difficulty. The eggs hatch rapidly, and the larvae can start feeding after $120 \mathrm{~h}$ of fertilization indicating the onset of experiments on zebrafish larvae from that point [17]. Another advantage is that the embryos are transparent, and all cells are observable since early larval stages. In addition, organs and tissues may also be readily visualized in vivo and can be examined instantly $[18,19]$. Furthermore, zebrafish is known to possess high fecundity rate generating large number of embryos. As an example, the females spawn around 300 eggs per week under ultimate conditions. This may result in fecundity of $>300,000$ eggs per $\mathrm{kg}$ of the female. Additionally, it can spawn in the laboratory aquarium by adding flora and gravel into the tank [20,21]. It has also been observed that the eggs hatch rapidly and organogenesis occurs quickly. As a result, the major organs are developed within 5-6 days post-fertilization (dpf) in larvae (Fig. 2). At the average of $350 \mathrm{dpf}$, females can attain size of $38 \mathrm{~mm}$ while males can attain a maximum mean size of $35 \mathrm{~mm}$ with a weight of 0.9 and $0.6 \mathrm{~g}$, respectively [22]. Another major advantage of zebrafish is that the cardiovascular, nervous and digestive systems of this model animal are similar to mammals [20]. In addition, highly conserved signaling pathways are found both in zebrafish and humans with a high level of genomic homology [23]. Thus, genetic analysis assessment of a particular gene function by transgenic development and knockdown experiments can also be performed through zebrafish with an ease [24]. Recently, National 


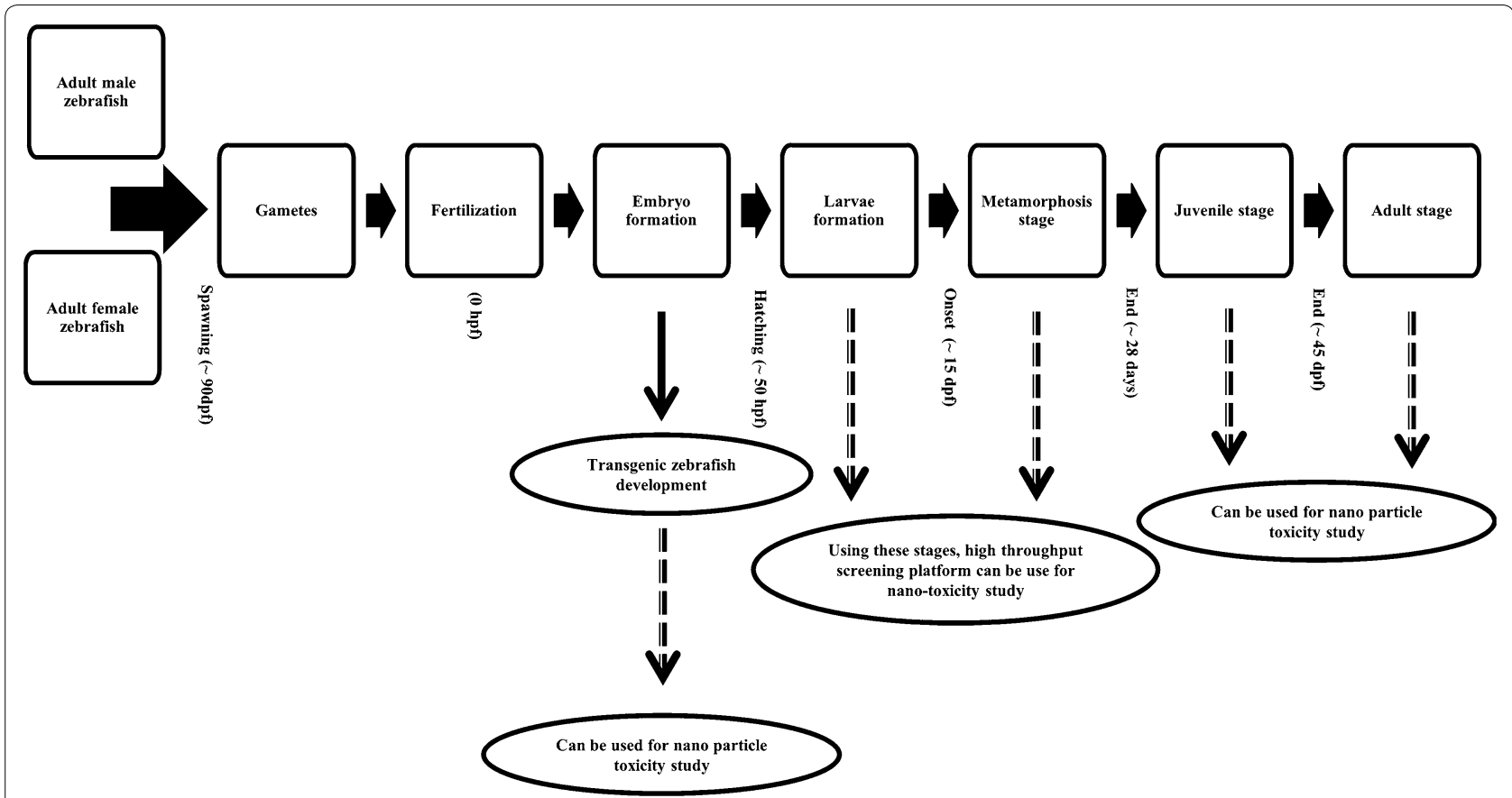

Fig. 2 Schematic diagram describes - the different stages of zebrafish development and their relevancy for nanotoxicity study

Institutes of Health (NIH), USA, has started to encourage the zebrafish model organism for the analysis of different diseases with a genetic program [25]. Zebrafish genetic map has also been developed, showing $>2000$ microsatellite markers and 400 distinct genes. As observed, high level of resemblance exists among the human and zebrafish genomes (more or less $75 \%$ similarity) making it a feasible animal model for analytical studies [26].

\section{Different model organisms and uniqueness of zebrafish model to understand the toxicity}

Several model organisms are been explored to understand the mechanism of various human diseases along with their genetic disorders. Frequently used organisms are yeast (Saccharomyces) [27], Drosophila [28], Caenorhabditis elegans (C. elegans) [29], zebrafish [30], mouse [31], monkey [32], and many more. Drosophila or $C$. elegans are outstanding models for studying the events of genetic functions related to the common molecular machine. These models help us to understand regulatory molecular machinery, such as a complex of communicating proteins or a signal-transduction pathway [33, 34]. On the contrary vertebrate model systems such as the mouse, zebrafish, and monkey are most preferred models for the human diseased state as compared to invertebrate model organisms. However, vertebrate model organisms are the most complex model systems among all of them. Both the advantages and disadvantages of all these animal model organisms are already discussed in previous reports $[35,36]$.

Among the entire vertebrate models the most widely studied is mouse model system. However in mouse model, some disadvantages are notable such as (1) "forward" genetics is difficult; (2) inside the mother embryonic manipulations and fetal experiments are complicated; (3) developmental stages, as well as life cycle, is moderately time-consuming; (4) high cost is involved for animal breeding and developing animal house facilities. As zebrafish offers various advantages over mouse model, it is emerging as an important model system which can connect development, disease, and toxicological studies. Henceforth, in the present scenario zebrafish can be represented as an interesting tool for assessing nanoparticle toxicity [20].

\section{Different assessment methods to evaluate nanoparticle toxicity}

In respect to various advantages offered by zebrafish, as discussed above, it is a unique model from the view point of the environment and human safety (EHS). The information produced from nano EHS studies can help to manage the risk of nanomaterials and nanotechnology related products in near future. The information may also assist us in framing the effective guidelines on protective measures, quality controls, and design strategies for improving nanomaterials and minimizing toxicity 
[37-39]. Using this model organism, several specific protocols have been used for the toxicity screening which are as follows:

\section{Hatching achievement analysis}

Zebrafish hatching is one of the most important events for the researchers to understand the chemical/nanomaterial toxicity. Various reports described the time span of zebrafish embryonic developmental stages, in detail. Acceding to Kimmel et al. [40] hatching occurs within the first 3 days. Furthermore, Villamizar et al. [41] illustrated that hatching event is related to a rhythmic pattern corresponding to the light phase. The maximum eggs are hatched at approximately $2 \mathrm{dpf}$ and the left over hatches roughly at $3 \mathrm{dpf}$. The correlation of successful hatching efficiency and embryo toxicity is an important parameter to evaluate the nanotoxicity. Recently, researchers used $\mathrm{TiO}_{2}$ nano-particles to understand the hatching events and the embryo toxicity by evaluating the relationship between hatching success rate and hours post exposure. It was observed that $\mathrm{TiO} 2$ nanoparticles can cause premature hatching in zebrafish embryos, dose dependently [42]. Ong et al. [43] also presented a view insight of zebrafish hatching under the effect of nanoparticle. They reported complete inhibition of hatching and embryo death within chorion upon nanoparticle exposure. They also concluded that nanoparticles interact with the hatching enzymes and thus they are responsible for toxicity rather than their ionic forms. Also, Vogt et al. [44] analyzed the chemical toxicity using zebrafish embryos at 24-48 $\mathrm{h}$ post fertilization (HPF) in 96-well plates. In this assay, multi-well plates are used with zebrafish embryos with the addition of a small molecule (BCI) to analyze hyper-activation of FGF signaling .

\section{Developmental malformation analysis of embryos and organs}

Malformation of embryos and organs is another parameter for toxicity screening. Developmental malformation includes incomplete organ development such as eye or incomplete body part development such as incomplete head formation and deformities of body parts such as bent notochord, fin malformation and lack of pigmentation. Ali and Legler [45] showed the non-lethal developmental malformations of zebrafish embryos after the exposure of chemical (nonylphenol) even at low dose. Moreover, Usenko et al. [46] evaluated of carbon fullerene $\left[\mathrm{C}_{60}, \mathrm{C}_{70}\right.$, and $\left.\mathrm{C}_{60}(\mathrm{OH})_{24}\right]$ toxicity using zebrafish embryos, in vivo. They observed caudal fin malformation at the concentrations of $200 \mathrm{ppb}$ of $\mathrm{C}_{60}, \mathrm{C}_{70}$ and yolk sac edema, pericardial edema and pectoral fin malformations over the concentrations of $2500 \mathrm{ppb}$ of $\mathrm{C}_{60}(\mathrm{OH})_{24}$. Additionally, they also observed swelling of zebrafish embryo and delay in development upon exposure to $5000 \mathrm{ppb}$ of $\mathrm{C}_{60}(\mathrm{OH})_{24}$. Therefore, the use of zebrafish model can be proposed for screening the toxicity profile of nanomaterials and their rapid feedback.

\section{In vitro/in vivo imaging}

Another important use of this model is to analyze the toxicity through in vitro imaging. Several specific types of imaging techniques can be used for toxicity study. For instance, dynamical cell imaging of zebrafish embryos is a method for safety assessment which is now used by several scientists [47]. Additionally, high-content imaging (HCI) methods also permit us to collect automated visual data as well as image analysis. The $\mathrm{HCI}$ method is very rapid and can be used for the chemical screening in zebrafish larvae [48]. Presently, whole-body imaging is a challenging method to understand the pathophysiology of 3D morphological structures. Using synchrotron $\mathrm{X}$-ray micro-CT, whole-body imaging was performed for hypercholesterolemic female zebrafish to understand the pathophysiology of 3D morphological structures [49]. Furthermore, in vivo real-time imaging is also used to understand the toxicity as well as size-dependent transport of metal nanoparticles using zebrafish embryos. Although the commercialization of silver (Ag) nanoparticles is expanding, its toxicity is also well known [50]. In view of this, recently a bio-imaging study on the toxic effect of sodium cholate templated Ag nanoclusters during the developmental stages of zebrafish embryo was conducted [51]. Thus, due to the wide application of bioimaging, zebrafish can be further explored to determine the toxicity profile of various nanomaterials.

\section{Transgenic zebrafish as live biosensor}

Transgenic zebrafish model is a potential choice in toxicological studies and currently, it is also used to evaluate the toxicity as live biosensor (Table 1). After exposed to toxic chemicals, morphological changes are well noticeable in zebrafish [52]. Lee et al. [53] developed a transgenic line of zebrafish embryos entitled as "huORFZ embryos" which can accurately detect various kinds of pollutants compounds and can be further used as water alarm system to monitor the disposal of hazardous pollutants in the water bodies. The significance of cytochrome P450 (CYPs) as an important biomarker for detecting carcinogen compounds, such as polycyclic aromatic hydrocarbons (PAHs), is well reported. Therefore, Hung et al. [54] generated a transgenic line of zebrafish using a CYPgreen fluorescence protein (CYP-GFP) construct for live imaging and to detect the water toxicity level using polychlorinated biphenyls (PCBs) as pollutant. They observed various morphological alterations in zebrafish upon PCB exposure suggesting the use of CYP-GFP as a live 


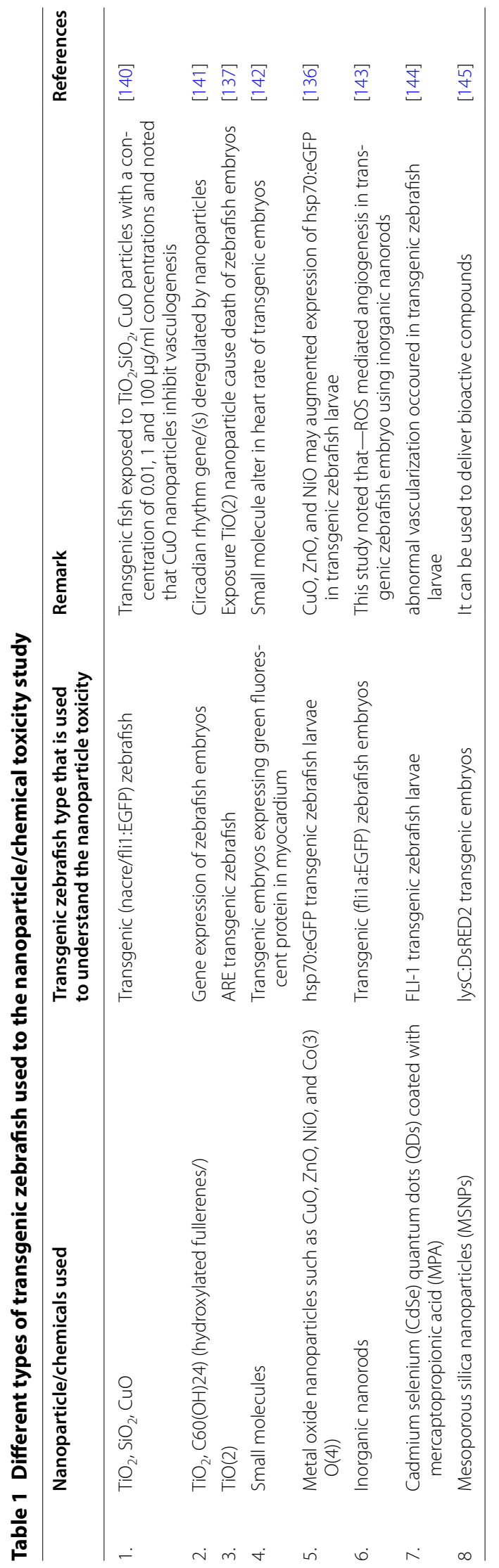


biosensor. Almeida et al. [55] also developed fluorescent transgenic zebrafish expressing a destabilized fluorescent protein (DsRED) to understand the toxicity generated by pesticide i.e. methyl parathione. Their observations also suggested the use of transgenic zebrafish as a potential live biomarker for toxicity analysis.

\section{Behavioral analysis}

Interestingly, zebrafish behavioral response is also a sensitive indicator for abnormal change in toxicity and thus nowadays it has become a most important parameter to estimate the toxicity level [56]. Within the behavioral responses, swimming kinetics is the most relevant and highly studied parameter. It has been prominently recorded that swimming speed and depth were altered by the chemical toxicity [57]. Kokel et al. [58] generated a behavioral 'barcode' to understand the chemical toxicity. Behavioral abnormalities such as abnormal startle behavior following a tap stimulus after exposure to gold nanoparticles at $122 \mathrm{dpf}$ was evaluated by Truong et al. [59]. Another experiment performed by Chen et al. [60] has also shown that $\mathrm{TiO} 2$ nanoparticles affect larval swimming parameters, including velocity and activity level.

\section{Disruption of gill, skin and endocrine system}

The disruption of gills, skin and endocrine system by nanoparticles is another parameter to understand nanoparticle induced toxicity. It has been reported that gills are most important targets of waterborne objects such as waterborne nanoparticle. Silver ions $\left(\mathrm{Ag}^{+}\right)$generated by Ag nanoparticles are well documented for their acute toxicity, mainly due to their interaction with the gills. In the gills, ionic $\mathrm{Ag}^{+}$inhibits $\mathrm{Na}^{+} / \mathrm{K}^{+}$-ATPase action and the enzymes related to $\mathrm{Na}^{+}$and $\mathrm{Cl}^{-}$uptake, finally affecting osmoregulation [61, 62]. Griffitt et al. [63] showed that $\mathrm{Cu}$ nanoparticles (insoluble forms) are extremely toxic to zebrafish and their suspensions may damage gills lamellae. In addition reports showed that nanoparticle are also toxic to zebrafish skin. Researchers have observed deposition in zebrafish skin upon exposure to nanoparticle like Ag-BSA. In the embryo skin, the nanoparticles enter through diffusion or endocytosis where they accumulate on the epidermis layer of the larvae and causes skin abnormalities through apoptosis [64]. Chemical exposure can also cause endocrine disruption in zebrafish. Tu et al. [65] described the endocrine disruption in zebrafish by chemicals and performed endocrine gene transcription analysis which resulted in the increased expression of the estrogen-responsive gene Vtg1. The results also showed that there is no effect on the expression of the ER $\alpha$ gene. Another experiment by Miao et al. [66] reported that the titanium dioxide nanoparticles increased the bioconcentration of lead, and directs the interruption of thyroid endocrine system in larvae of zebrafish.

\section{Immunotoxicity}

Immunotoxicity is classified as the toxic effect of xenobiotics on the normal functioning of immune system through direct or indirect methods. Direct immunotoxicity causes immune suppression leading to reduced resistance against various diseases, such as cancer [67]. Immunotoxicity of nanoparticles has been demonstrated by several scientists. Various studies showed that nanoparticles (including metal oxide nanoparticles) modulate cytokine production by generating free radicals. Some nanoparticles have also been linked to allergic sensitization and can increase the tendency of asthma [68]. Jin and Zheng [69] reported that a toxic chemical like cypermethrin induces apoptosis and immunotoxicity in zebrafish (Danio rerio) suggesting the possible use of zebrafish in immunotoxicity studies. Furthermore, Zhuang et al. [70] described the enantio-selective differences in the developmental toxicity and immunotoxicity of pyraclofoson zebrafish model. Several reports also suggested the use of live zebrafish embryos for analyzing the immunotoxic property of various chemicals. In this regard, Xu et al. [71] used transgenic, albino or $\mathrm{AB}$ line, live zebrafish embryos to understand the immune-toxic effects of chemicals such as dibutylphthalate. However, more researches are needed on nanoparticle-based immunotoxicity analysis on zebrafish.

\section{Genotoxicity}

Genotoxicity is described as the damage caused to genetic information inside a cell due to the occurrence of chemical agents which results in gene mutation, chromosomal alteration and DNA damage [72]. Genotoxicity is a main risk factor for long-term toxic effects such as carcinogenesis. Zebrafish model has been proposed to study the different chemical-induced genotoxicity using various techniques. Cambier et al. [73] studied genotoxic effects of cadmium on zebrafish through RAPD and RT PCR. Furthermore, genotoxic effects of gold nanoparticle were also assessed using RAPD-based methodology on zebrafish after exposure to gold NPs [74]. In accordance to the above mentioned result, Geffroy et al. [75] investigated the effects of nanoparticles on genotoxicity through RAPD-PCR genotoxicity test in the zebrafish at very low doses of gold NP (36-106 ng gold/fish/day) and resulted in significant alteration of genome composition. However, to date fewer reports are available for genotoxic analysis of nanoparticles on zebrafish and thus more extensive studies are required in this area. 


\section{Neurotoxicity}

Due to the contact with toxic substances, the nervous tissue gets damaged resulting in significant irregular activity of the nervous system, which is called neurotoxicity. These toxic substances to nerve cell are referred as neurotoxins [76]. Neurotoxicity of nanoparticles has been determined earlier from time to time suggesting that nanoparticles can reach the brain and can cause neurodegeneration $[77,78]$. In vivo and in vitro experiments discovered that combustion-derived nanoparticles are neurotoxic, because of the incidence of nanoparticle aggregation [79]. Therefore, in view of applying zebrafish model to determine nanoparticle neurotoxicity the radioprotective effect of dendrofullerene nanoparticle (DF-1), a $\mathrm{C}_{60}$ fullerene derivative, has been assessed in zebrafish embryo to understand the neurotoxicity resulting in dose-limiting toxicity level [80].

Sheng et al. [81] also evaluated $\mathrm{TiO}_{2}$ nanoparticleinduced neurotoxicity using zebrafish model. It has been reported that $\mathrm{TiO}_{2}$ nanoparticle significantly activated expressions of different genes such as BDNF C-fos and $\mathrm{C}$-jun. Conversely, this NP suppressed the expressions of genes such as p38, NGF, CRE resulting in the brain damage of zebrafish. Such contradictory observations provide a requirement for evaluating the nanoparticle caused neurotoxicity in future studies.

\section{Reproductive toxicity}

Reproductive toxicity is also among the important parameters of nanoparticle toxicity measurement [82]. Zebrafish model is one of the best models to assess the reproductive toxicity due to its high reproduction rate. As observed, nanoparticle affects male and female reproductivity and fetal development. Wang et al. [83] assessed the disturbance in zebrafish reproduction after the chronic exposure of $\mathrm{TiO}_{2}$ nanoparticles. Their study showed $9.5 \%$ decrease in the collective number of zebrafish eggs after 13 weeks of $\mathrm{TiO}_{2}$ nanoparticle exposure.

\section{Mortality assessment}

Acute exposure to nanoparticles enhances the chance of critical illness as well as increase in mortality rate. Duan et al. [84] treated zebrafish embryos with silica nanoparticles in their experimental model system and observed enhanced embryonic mortality. $\mathrm{ZnO}$ is another toxic nanoparticles which caused hatching delay, skin ulceration and high mortality of zebrafish [85]. It has been illustrated that a relationship exists between the shape of the nanoparticles and the mortality incidences. Therefore, Hua et al. [86] evaluated the toxic effects of differently shaped zinc oxide nanoparticles and observed that
$\mathrm{ZnO}$ nanosticks are more toxic as compare to other NPs resulting in increased mortality and reduced hatching in zebrafish. Polyamidoamine (PAMAM) is one of the significant dendrimers containing a diamine which is used as versatile precursor for dendrimer-based delivery of active pharmaceuticals [87]. Pryor et al. [88] showed $100 \%$ mortality in zebrafish at 24 hpf by PAMAM dendrimers illustrating its toxic effects.

\section{Other methods}

Chemical toxicity screening in zebrafish model has been performed using both larval stage as well as adult zebrafish [89]. Hermsen et al. [90] developed zebrafish embryo toxicity (ZET) test to analyze embryo- toxicity caused by two classes of chemicals in a modified zebrafish embryo. In their study, toxicity level of six 1,2,4-triazole antifungals and eight glycol ethers were evaluated. Among glycol ether metabolites methoxyacetic acid and ethoxyacetic acid and among triazoles flusilazole were most potent in retarding the growth and inducing malformation in zebrafish. Another newly developed computational analysis has also been used by Toxicology Research Program of the U.S. EPA to understand the toxicity of the chemical library. Through the experiments, researchers evaluated the toxicity of the 309 ToxCast ${ }^{\mathrm{TM}}$ Phase I chemicals using a zebrafish screen [91]. In a study, whole male adult zebrafish was exposed to high doses of nickel chloride, cobalt chloride or sodium dichromate for $24 \mathrm{~h}$ at various concentrations corresponding to their respective $96 \mathrm{~h} \mathrm{LC}$ values and was evaluated for alterations in gene expressions. Histopathological changes were observed for each metal in their target specific organs and tissues signifying the role of adult zebrafish as a robust model in toxicogenomics [92].

\section{Nanotoxicology in zebrafish}

Nanotoxicology is an interdisciplinary field, cross-linking various subjects such as chemistry, physics, biology, medicine and toxicology [93, 94]. Though, this area has many applicative approaches yet it is still in nascent form and the majority of the nanotoxicology studies are confined only within in vitro models. Some research groups performed toxicological studies using animal models (in vivo) which are supposed to be significant due to the diversity in animal physiology and anatomy in animal models. Recently, for this purpose zebrafish is proposed as one of the most successful model and notable advancement has been made in nanotoxicology studies using zebrafish as animal model [95]. This section categorizes some of the currently available toxicity data of several nanoparticles which has been studied using zebrafish model. 


\section{Silver nanoparticle}

Silver nanoparticles are the most extensively studied nanoparticles. They are also widely used as therapeutic agents [96], as antimicrobial agents [97], in drug delivery systems [98], as biosensors [99] and in cosmetics [100]. Size-dependent toxicity of AgNP was observed indicating that the size of the NP is one of the important factors for their toxicity profile. Lee et al. [101] performed in vivo quantitative study and demonstrated sizedependent transport and toxicity of Ag-NPs in zebrafish. In their study, they showed that Ag nanoparticle in size range $30-72 \mathrm{~nm}$ diameters were able to diffuse into the zebrafish embryos through chorionic pores via random Brownian motion and thus they can show more potent toxic effect. On the contrary, Bar-Ilan et al. [102] synthesized AgNP in different size range (3, 10, 50 and $200 \mathrm{~nm}$ ) and treated them to zebrafish embryos rearing container. They observed that there were size independent $100 \%$ mortality incidences after $120 \mathrm{hpf}$. Hence, the size dependent toxicity profile of AgNP is still debatable. Surface defect is another parameter of nanoparticle toxicity. George et al. [103] verified the toxic effect and surface defect caused by Ag nanoparticle in both fish cell lines and zebrafish embryos. Furthermore, in a recent study George et al. [104] also showed the effect of solar light in increasing the toxicity of Ag.

The charge dependent transport and toxicity of peptide-functionalized Ag-NPs into early developing stage zebrafish embryos was also demonstrated. A recent study concluded that Ag-peptide NPs are much more biocompatible than the citrated Ag NPs [105]. Choi et al. [106] evaluated oxidative stress and apoptosis in zebrafish liver and concluded the hepatotoxic behavior of AgNPs. They also observed the disruption of hepatic cell cords and apoptotic changes in Ag nanoparticles exposed zebrafish due to the upregulation of p53-related pro-apoptotic genes such Bax, p21 and Noxa. The influence of AgNPs on the neurological development of zebrafish was studied by Xin et al. [107].They showed that the introduction to AgNPs can alter the neurological development and can result in small head along with the presence of hypoplastic hind brain, little eye, and cardiac defects [107]. In view of the toxicity profile of AgNP, some recent studies proposed that the chemical transformations of Ag nanoparticles can be an important way to slow down the nanoparticle toxicity. For instance, Devi et al. [108] proved that sulfidation method can delay AgNP toxicity in zebrafish model. Furthermore, biosynthesis of silver nanoparticles can also be a better way to reduce its toxicity level [109].

\section{Gold nanoparticle}

Nanoscale gold particles have attained massive scientific interest due to their chemical stability and unique optical properties [110]. Gold nanoparticles have shown great potential in medical industry such as therapeutic agents, as photothermal therapeutic agent, as drug carriers, etc. [111]. Presently, gold nanoparticles are used as promising agents for cancer therapy [112], as imaging particles in optical microscopy [113] and confocal laser microscopy [114]. It is also used in diagnostics such as dot immunoassay, immunochromatography, etc. [115]. Today, gold nanoparticles are among the most widely studied nanoparticles.

Like other metal nanoparticle, cytotoxicity of gold nanoparticles in humans has also been reported $[116,117]$. Therefore, to understand the in vivo toxicity profile of gold nanoparticles some studies used zebrafish as in vivo animal model. Kim et al. [118] analyzed the toxicity outcomes of cationic ligand functionalized gold nanoparticles in the development of zebrafish embryos including lethality and other morphological defects. As observed, the functionalized gold particles caused abnormalities in the eye development and affected pigmentation in eyes leading to behavioral and neuronal damage. Harper et al. [119] assessed the toxicity mapping of AuNPs of different sizes and surface charges (such as neutral, positive and negative surface charges) using an embryonic zebrafish model. They concluded that the mortality and other developmental disorders are closely related to the morphological and chemical characteristics of gold nanoparticles signifying of the importance of controlled synthesis of nanomaterials. In another similar approach, toxic effect of gold nanoparticles possessing three different functional group with different surface charges on zebrafish embryos was assessed exhibiting hypo-locomotor activity and abnormal behavioral activity [59]. Real time in vivo imaging was further used in a different study to determine the size-dependent transport and toxicity of AuNPs in zebrafish embryos which revealed the presence of AuNPs inside the embryos throughout their whole developmental duration [50]. In addition, Dedeh et al. [74] exposed zebrafish with AuNPs for 20 days at two concentrations i.e. 16 and $55 \mu \mathrm{g} / \mathrm{g}$ dry weights and recorded the alterations in oxidative stress, mitochondrial metabolism and neurotransmission.

\section{Carbon nanotubes (CNT)}

Carbon nanotube showed numerous distinctive chemical and physical characteristics which make it equivalent to the biological macromolecules such as antibodies, enzymes, plasmid DNA, etc. CNTs have become a promising candidate for the delivery of chemotherapeutic agents such as paclitaxel and doxorubicin, small interfering RNA (siRNA), different biological molecules, genes, vaccines and antibodies. This is due to the fact that because of high surface area it can conjugate with a wide 
variety of therapeutic molecules [120, 121]. However, the toxicity of carbon nanotube is an upcoming and significant challenge nowadays [122]. The toxicity reports explaining the potential impact of CNT's on human health and the environment are very inadequate and thus it requires more studies through improved methodologies. Ali-Boucetta et al. [123] discussed the pitfalls in traditionally used in vitro models for toxicological studies and highlighted the need for more reliable model systems. They also performed MTT and the LDH assays to comprehend the interaction of CNT with cell cultures and to understand the cytotoxicity of CNT's. Luanpitpong et al. [124] described the effects of CNT on lung and dermal cellular behaviors which showed the potential pulmonary and dermal risk associated with CNT exposure.

Recently, using zebrafish model bioaccumulation and distribution of multiwalled CNTs was evaluated which showed the bioaccumulation factor of $16 \mathrm{~L} / \mathrm{kg}$ fish wet weight [125]. Another study performed by $\mathrm{Li}$ et al. [126] revealed CNT-induced biochemical alterations in zebrafish. They further demonstrated that CNT exposures can stimulate the brain and gonadal alterations. In a different study, the toxicity level of functionalized CNT's with different lengths was evaluated in zebrafish embryo and was further suggested that length of CNT's also plays a major role in their toxicity profile, in vivo [127]. In a study by FilhoJde et al. [128] CNT network pellets having agglomerated multi-walled CNTs (MWCNTs) with a standard diameter about $500 \mathrm{~nm}$ were not found genotoxic to zebrafish model. However, they observed reversible inflammation in the zebrafish gills.

Besides MWCNTs, the malformations and mortality induced by $\mathrm{C}_{60}$ fullerene was also studies in zebrafish embryo. It was observed that $\mathrm{C}_{60}$ fullerene causes dose dependent necrotic and apoptotic cell death in the head and trunk of zebrafish embryo. Although in comparison to $\mathrm{C}_{60}, \mathrm{C}_{60}(\mathrm{OH})_{24}$ was found less toxic to the head region. Further, it was demonstrated that $\mathrm{C}_{60}$ causes oxidative stress and was depicted as the main reason for malformations in zebrafish embryo [46].

\section{Metal oxide nanoparticles}

Metal oxide nanomaterials are commercially used in different emerging areas such as information technology, energy storage, medicine, and catalysis $[129,130]$. Several types of metal oxide nanoparticles are used in industrial production of nanoparticles such as $\mathrm{TiO}_{2}$, $\mathrm{ZnO}, \mathrm{Fe}_{3} \mathrm{O}_{4}, \mathrm{Al}_{2} \mathrm{O}_{3}$, and $\mathrm{CrO}_{3}$. Jeng and Swanson examined the toxicity of metal oxide nanoparticles where they observed apoptotic behavior of $\mathrm{ZnO}$ nanoparticles in cells [131]. The toxicity of metal oxide nanoparticles was also assessed through zebrafish model. Zhu et al. [132] evaluated the toxicity of three metal oxide nanoparticles $\left(\mathrm{TiO}_{2}, \mathrm{Al}_{2} \mathrm{O}_{3}\right.$ and $\left.\mathrm{ZnO}\right)$ using zebrafish embryos. They observed tissue ulceration in zebrafish larvae exposed to aqueous suspensions of $\mathrm{nZnO}$ and $\mathrm{ZnO}$ /bulk. Additionally, they showed that $\mathrm{nTiO}_{2}, \mathrm{nZnO}$ and $\mathrm{nAl}_{2} \mathrm{O}_{3}$ exerted toxic effects on both zebrafish embryos and larvae. An attractive study was performed by Palaniappan and Pramod to compare the effect of bulk and nano $\mathrm{TiO}_{2}$ on zebrafish brain by using FT-IR technique where $\mathrm{nTiO}_{2}$ proved to be more toxic as compared to bulk $\mathrm{TiO}_{2}$ [133]. Similar results were again observed by Palaniappan and Pramod while investigating the effect of $\mathrm{nTiO}_{2}$ and bulk $\mathrm{TiO}_{2}$ on zebrafish by FT-Raman Spectroscopy [134]. Additionally, Chen et al. [135] also explored that nano $\mathrm{TiO}_{2}$ causes toxicity effects to zebrafish model, and the chronic toxicity of $\mathrm{TiO}_{2} \mathrm{NP}$ is concentration as well as time dependent. These nanoparticles get distributed and accumulate in different body parts of zebrafish such as gill, liver, heart and brain. Compared to other nanoparticle, $\mathrm{TiO}_{2}$ NPs can also cross blood-brain barrier [78]. Transgenic zebrafish is an important line which helps to detect the metal oxide toxicity. It has been observed that some other dissolvable metal oxide nanoparticles such as $\mathrm{CuO}, \mathrm{ZnO}$ and $\mathrm{NiO}$ hinders in hatching process of transgenic zebrafish embryos line generated using hsp70 and eGFP genes [136]. Bar-Ilan et al. [137] used a transgenic zebrafish line (ARE:eGFP) for detecting the generation of oxidative stress in the larvae upon $\mathrm{TiO}_{2}$ nanoparticles exposure. The experiment explains that $\mathrm{TiO}_{2} \mathrm{NPs}$ absorbs photons and produce electron-hole pairs that interact with water and oxygen to form cytotoxic ROS. Finally, researchers concluded that the $\mathrm{TiO}_{2}$ NPs exposure to zebrafish embryos leads to malformation and death.

\section{Drawbacks of zebrafish model for nanotoxicity study}

To evaluate the toxicity profile of nanomaterials, zebrafish model was used in various studies as an in vivo model system. The toxicity level of these nanoparticles was assessed by observing the malformations and functional defects in zebrafish. However, it is very clear from the literature survey that nanomaterial based immunotoxicity assay is still lacking. Additionally, due to the rapid developmental stages in zebrafish it is very difficult to perform systematic embryo-based nano-toxicity assays. However, automation and advanced technologies can help in nano-toxicity screening with zebrafish embryos. Several nanomaterials are used for the therapeutic purpose such as drug delivery and antimicrobial therapeutics. Therefore, it is needed to understand the absorption, distribution, metabolism and excretion (ADME) properties of these nanomaterials. However, it is ambiguous that 
how ADME assay will perform in zebrafish model after nano-drug delivery.

\section{Future prospects}

Zebrafish has shown its enormous potential as an in vivo model for nanomaterial toxicity study. Presently, various molecular biology techniques and zebrafish model transgenic lines have been developed for this purpose. Various zebrafish microarrays and huge genomic resources are nowadays available for nanotoxicity evaluation. All these advance resources makes zebrafish an extremely multipurpose system for toxicogenomic studies of nanomaterial in the very near future. Proteins and genes expression studies of zebrafish development have the huge possibility to uncover the still debated nanomaterial toxicity. Although, high throughput screening systems using larval stages of zebrafish is already being exploited for nanomaterial toxicity study, there is still an enormous potential for nanomaterial toxicity assays.

\section{Conclusion}

Presently, zebrafish have become a smart vertebrate model for toxicological testing. In Germany, the zebrafish embryo test was introduced as a standardized ISO program in the evaluation of chemicals. This assay can be used for water testing to evaluate the level of environmental contaminants [138]. Furthermore, this animal model is much faster, cheaper and more efficient animal model for more than a decade [139], and this model is used for toxicological testing of nanomaterial. With the use of modern technology, the zebrafish might be able to become a significant alternative of other mammalian models for toxicological testing of nanomaterial in forthcoming years.

\section{Abbreviations}

hsp70: heat shock protein 70; eGFP: enhanced green fluorescence protein: ROS: reactive oxygen species; DsRED: zebrafish transgenic lines that express green or red fluorescent proteins; lysC: lysozyme C; FLI-1 gene: friend leukemia integration 1 gene.

\section{Authors' contributions}

CC, ARS and GS collected the data and drafted the manuscript. ARS and SSL revised and edited the manuscript. All authors read and approved the final manuscript.

\begin{abstract}
Acknowledgements
This research was supported by Hallym University Research Fund, by Basic Science Research Program through the National Research Foundation of Korea (NRF) funded by the Ministry of Education (2014R1A1A4A03009388), and by a grant of the Korea Health Technology R\&D Project through the Korea Health Industry Development Institute (KHIDI), funded by the Ministry of Health \& Welfare (HI12C1265), Republic of Korea.
\end{abstract}

\section{Competing interests}

The authors declare that they have no competing interests.
Received: 10 May 2016 Accepted: 5 August 2016

Published online: 20 August 2016

References

1. Mangematin $\mathrm{V}$, Walsh $\mathrm{S}$. The future of nanotechnologies. Technovation. 2012;32:157-60.

2. Salamanca-Buentello F, Persad DL, Court EB, Martin DK, Daar AS, Singer PA. Nanotechnology and the developing world. PLoS Med. 2005;2:e97.

3. Sabourin V, Ayande A. Commercial opportunities and market demand for nanotechnologies in agribusiness sector. J Technol Manag Innov. 2015;10:40-51.

4. Freitas RA Jr. Welcome to the future of medicine. Stud Health Technol Inform. 2009;149:251-6.

5. Hyungsub C, Mody CCM. The long history of molecular electronics: microelectronics origins of nanotechnology. Soc Stud Sci. 2009:39:11-50.

6. Roco MC. Environmentally responsible development of nanotechnology. Environ Sci Technol. 2005;39:106A-12A.

7. Vance ME, Kuiken T, Vejerano EP, McGinnis SP, Hochella MF Jr, Rejeski D, Hull MS. Nanotechnology in the real world: redeveloping the nanomaterial consumer products inventory. Beilstein J Nanotechnol. 2015;6:1769-80.

8. Thanh NTK, Green LAW. Functionalization of nanoparticles for biomedical applications. Nano Today. 2010;5:213-30.

9. Xu L, Liu Y, Chen Z, Li W, Wang L, Wu X, Ji Y, Zhao Y, Ma L, Shao Y, Chen C. Surface-engineered gold nanorods: promising DNA vaccine adjuvant for HIV-1 treatment. Nano Lett. 2012;12:2003-12.

10. Tsuzuki T. Commercial-scale production of nanoparticles. Boca Raton: CRC Press Taylor \& Francis Group; 2013. p. 978-81.

11. Maynard AD, Warheit DB, Philbert MA. The new toxicology of sophisticated materials: nanotoxicology and beyond. Toxicol Sci. 2011;120(Suppl 1):S109-29.

12. Seaton A, Tran L, Aitken R, Donaldson K. Nanoparticles, human health hazard and regulation. J R Soc Interface. 2010;7(Suppl 1):S119-29.

13. Gambardella C, Gallus L, Gatti AM, Faimali M, Carbone S, Antisari LV. Toxicity and transfer of metal oxide nanoparticles from microalgae to sea urchin larvae. Chem Ecol. 2014;30:308-16.

14. Gad SC. Animal models in toxicology. London: CRC Press; 2014. p. 983.

15. Strahle U, Scholz S, Geisler R, Greiner P, Hollert H, Rastegar S, Schumacher A, Selderslaghs I, Weiss C, Witters H, BraunbeckT. Zebrafish embryos as an alternative to animal experiments-a commentary on the definition of the onset of protected life stages in animal welfare regulations. Reprod Toxicol. 2012;33:128-32.

16. Chakraborty C, Agoramoorthy G. Why zebrafish? Riv Biol. 2010;103:25-7.

17. Easter SS Jr, Nicola GN. The development of vision in the zebrafish (Danio rerio). Dev Biol. 1996;180:646-63.

18. Fishman MC. Zebrafish genetics: the enigma of arrival. Proc Natl Acad Sci USA. 1999;96:10554-6.

19. Parichy DM, Elizondo MR, Mills MG, Gordon TN, Engeszer RE. Normal table of post-embryonic zebrafish development: staging by externally visible anatomy of the living fish. Dev Dyn. 2009;238:2975-3015.

20. Hsu CH, Wen ZH, Lin CS, Chakraborty C. The zebrafish model: use in studying cellular mechanisms for a spectrum of clinical disease entities. Curr Neurovasc Res. 2007;4:111-20.

21. Spence R, Ashton R, Smith C. Oviposition decisions are mediated by spawning site quality in wild and domesticated zebrafish, Danio rerio. Behaviour. 2007:144:953-66.

22. Ribas L, Piferrer F. The zebrafish (Danio rerio) as a model organism, with emphasis on applications for finfish aquaculture research. Rev Aquacult. 2014;6:209-40.

23. Beliaeva NF, Kashirtseva VN, Medvedeva NV, Khudoklinova I, Ipatova OM, Archakov Al. Zebrafish as a model organism for biomedical studies. Biomed Khim. 2010;56:120-31.

24. Varshney GK, Lu J, Gildea DE, Huang H, Pei W, Yang Z, Huang SC, Schoenfeld D, Pho NH, Casero D, et al. A large-scale zebrafish gene knockout resource for the genome-wide study of gene function. Genome Res. 2013;23:727-35. 
25. Rasooly RS, Henken D, Freeman N, Tompkins L, Badman D, Briggs J, Hewitt AT. Genetic and genomic tools for zebrafish research: the NIH zebrafish initiative. Dev Dyn. 2003;228:490-6.

26. Chakraborty C, Hsu CH, Wen ZH, Lin CS, Agoramoorthy G. Zebrafish: a complete animal model for in vivo drug discovery and development. Curr Drug Metab. 2009;10:116-24.

27. Delorme-Axford E, Guimaraes RS, Reggiori F, Klionsky DJ. The yeast Saccharomyces cerevisiae: an overview of methods to study autophagy progression. Methods. 2015;75:3-12.

28. Foriel S, Willems P, Smeitink J, Schenck A, Beyrath J. Mitochondrial diseases: Drosophila melanogaster as a model to evaluate potential therapeutics. Int J Biochem Cell Biol. 2015;63:60-5.

29. Gonalez-Moragas L, Roig A, Laromaine A. C. elegans as a tool for in vivo nanoparticle assessment. Adv Colloid Interface Sci. 2015;219:10-26.

30. Montazerolghaem M, Nystrom L, Engqvist H, Karlsson Ott M. Zebrafish: A possible tool to evaluate bioactive ions. Acta Biomater. 2015;19:10-4.

31. Iguchi Y, Michiue H, Kitamatsu M, Hayashi Y, Takenaka F, Nishiki T, Matsui H. Tumor-specific delivery of BSH-3R for boron neutron capture therapy and positron emission tomography imaging in a mouse brain tumor model. Biomaterials. 2015;56:10-7.

32. Riccio EK, Pratt-Riccio LR, Bianco-Junior C, Sanchez V, Totino PR, Carvalho LJ, Daniel-Ribeiro CT. Molecular and immunological tools for the evaluation of the cellular immune response in the neotropical monkey Saimiri sciureus, a non-human primate model for malaria research. Malar J. 2015;14:166

33. Fernandes VM, Pradhan-Sundd T, Blaquiere JA, Verheyen EM. Ras/ MEK/MAPK-mediated regulation of heparin sulphate proteoglycans promotes retinal fate in the Drosophila eye-antennal disc. Dev Biol. 2015:402:109-18.

34. Pastuhov SI, Hisamoto N, Matsumoto K. MAP kinase cascades regulating axon regeneration in C. elegans. Proc Jpn Acad Ser B Phys Biol Sci. 2015;91:63-75.

35. Jucker $M$. The benefits and limitations of animal models for translational research in neurodegenerative diseases. Nat Med. 2010;16:1210-4.

36. Loisel S, Ohresser M, Pallardy M, Dayde D, Berthou C, Cartron G, Watier $H$. Relevance, advantages and limitations of animal models used in the development of monoclonal antibodies for cancer treatment. Crit Rev Oncol Hematol. 2007;62:34-42.

37. Kahru A, Dubourguier HC. From ecotoxicology to nanoecotoxicology. Toxicology. 2010;269:105-19.

38. Wang J, Asbach C, Fissan H, Hülser T, Kuhlbusch TAJ, Thompson D, Pui DYH. How can nanobiotechnology oversight advance science and industry: examples from environmental, health, and safety studies of nanoparticles (nano-EHS). J Nanoparticle Res. 2011:13:1373-87.

39. Youtie J, Porter A, Shapira P, Tang L, Benn T. The use of environmental, health and safety research in nanotechnology research. J Nanosci Nanotechnol. 2011;11:158-66.

40. Kimmel CB, Ballard WW, Kimmel SR, Ullmann B, Schilling TF. Stages of embryonic development of the zebrafish. Dev Dyn. 1995;203:253-310.

41. Villamizar N, Ribas L, Piferrer F, Vera LM, Sanchez-Vazquez FJ. Impact of daily thermocycles on hatching rhythms, larval performance and sex differentiation of zebrafish. PLOS ONE. 2012;7:e52153.

42. Samaee SM, Rabbani S, Jovanovic B, Mohajeri-Tehrani MR, Haghpanah $V$. Efficacy of the hatching event in assessing the embryo toxicity of the nano-sized $\mathrm{TiO}(2)$ particles in zebrafish: a comparison between two different classes of hatching-derived variables. Ecotoxicol Environ Saf. 2015;116:121-8

43. Ong KJ, Zhao X, Thistle ME, Maccormack TJ, Clark RJ, Ma G, MartinezRubi Y, Simard B, Loo JS, Veinot JG, Goss GG. Mechanistic insights into the effect of nanoparticles on zebrafish hatch. Nanotoxicology. 2014;8:295-304

44. Vogt A, Codore H, Day BW, Hukriede NA, Tsang M. Development of automated imaging and analysis for zebrafish chemical screens. J Vis Exp. 2010;40:e1900

45. Ali ES, Legler J. Developmental toxicity of nonylphenol in zebrafish(Danio rerio) embryos. Indian J Geomarine Sci. 2011;40:509-15.

46. Usenko CY, Harper SL, Tanguay RL. In vivo evaluation of carbon fullerene toxicity using embryonic zebrafish. Carbon N Y. 2007;45:1891-8.
47. Knudsen TB, Kavlock RJ, Daston GP, Stedman D, Hixon M, Kim JH. Developmental toxicity testing for safety assessment: new approaches and technologies. Birth Defects Res B Dev Reprod Toxicol. 2011;92:413-20.

48. Peravali R, Gehrig J, Giselbrecht S, Lutjohann DS, Hadzhiev Y, Muller F, Liebel U. Automated feature detection and imaging for high-resolution screening of zebrafish embryos. Biotechniques. 2011;50:319-24.

49. Seo E, Lim JH, Seo SJ, Lee SJ. Whole-body imaging of a hypercholesterolemic female zebrafish by using synchrotron X-ray micro-CT. Zebrafish. 2015;12:11-20.

50. Browning LM, Huang T, Xu XH. Real-time in vivo imaging of sizedependent transport and toxicity of gold nanoparticles in zebrafish embryos using single nanoparticle plasmonic spectroscopy. Interface Focus. 2013;3:20120098

51. Chandirasekar S, Chandrasekaran C, Muthukumarasamyvel T, Sudhandiran G, Rajendiran N. Sodium cholate-templated blue lightemitting Ag subnanoclusters: in vivo toxicity and imaging in zebrafish embryos. ACS Appl Mater Interfaces. 2015;7:1422-30.

52. Lee O, Green JM, Tyler CR. Transgenic fish systems and their application in ecotoxicology. Critical Rev Toxicol. 2015;45:124-41.

53. Lee HC, Lu PN, Huang HL, Chu C, Li HP, Tsai HJ. Zebrafish transgenic line huORFZ is an effective living bioindicator for detecting environmental toxicants. PLoS ONE. 2014:9:e90160.

54. Hung KW, Suen MF, Chen YF, Cai HB, Mo ZX, Yung KK. Detection of water toxicity using cytochrome P450 transgenic zebrafish as live biosensor: for polychlorinated biphenyls toxicity. Biosens Bioelectron. 2012;31:548-53.

55. Almeida DV, Vaz B, Azevedo Figueiredo M, Junior AS, Marins LF. Fluorescent transgenic zebrafish as a biosensor for growth-related effects of methyl parathion. Aquat Toxicol. 2014;152:147-51.

56. MacPhail RC, Hunter DL, Irons TD, Padilla S. Locomotion and behavioral toxicity in larval zebrafish: background, methods, and data. Zebrafish Methods Assess Drug Saf Tox. 2011:151-164.

57. Huang $Y$, Zhang J, Han X, Huang T. The use of zebrafish (Danio rerio) behavioral responses in identifying sublethal exposures to deltamethrin. Int J Environ Res Public Health. 2014;11:3650-60.

58. Kokel D, Bryan J, Laggner C, White R, Cheung CY, Mateus R, Healey D, Kim S, Werdich AA, Haggarty SJ, et al. Rapid behavior-based identification of neuroactive small molecules in the zebrafish. Nat Chem Biol. 2010;6:231-7

59. Truong L, Saili KS, Miller JM, Hutchison JE, Tanguay RL. Persistent adult zebrafish behavioral deficits results from acute embryonic exposure to gold nanoparticles. Comp Biochem Physiol C Toxicol Pharmacol. 2012;155:269-74.

60. Chen TH, Lin CY, Tseng MC. Behavioral effects of titanium dioxide nanoparticles on larval zebrafish (Danio rerio). Mar Pollut Bull. 2011;63:303-8.

61. Bury NR, Grosell M, Grover AK, Wood CM. ATP-dependent silver transport across the basolateral membrane of rainbow trout gills. Toxicol Appl Pharmacol. 1999;159:1-8.

62. Wood CM, Playle RC, Hogstrand C. Physiology and modeling of mechanisms of silver uptake and toxicity in fish. Environmental Toxicol Chem. 1999:18:71-83.

63. Griffitt RJ, Weil R, Hyndman KA, Denslow ND, Powers K, Taylor D, Barber DS. Exposure to copper nanoparticles causes gill injury and acute lethality in zebrafish (Danio rerio). Environ Sci Technol. 2007:41:8178-86.

64. Asharani PV, Lian Wu Y, Gong Z, Valiyaveettil S. Toxicity of silver nanoparticles in zebrafish models. Nanotechnology. 2008;19:255102.

65. Tu W, Niu L, Liu W, Xu C. Embryonic exposure to butachlor in zebrafish (Danio rerio): endocrine disruption, developmental toxicity and immunotoxicity. Ecotoxicol Environ Saf. 2013;89:189-95.

66. Miao W, Zhu B, Xiao X, Li Y, Dirbaba NB, Zhou B, Wu H. Effects of titanium dioxide nanoparticles on lead bioconcentration and toxicity on thyroid endocrine system and neuronal development in zebrafish larvae. Aquat Toxicol. 2015;161:117-26.

67. Lankveld DP, Van Loveren H, Baken KA, Vandebriel RJ. In vitro testing for direct immunotoxicity: state of the art. Methods Mol Biol. 2010:598:401-23.

68. Di Gioacchino M, Petrarca C, Lazzarin F, Di Giampaolo L, Sabbioni E, Boscolo P, Mariani-Costantini R, Bernardini G. Immunotoxicity of nanoparticles. Int J Immunopathol Pharmacol. 2011;24:65S-71S. 
69. Jin Y, Zheng S, Fu Z. Embryonic exposure to cypermethrin induces apoptosis and immunotoxicity in zebrafish (Danio rerio). Fish Shellfish Immunol. 2011;30:1049-54.

70. Zhuang S, Zhang Z, Zhang W, Bao L, Xu C, Zhang H. Enantioselective developmental toxicity and immunotoxicity of pyraclofos toward zebrafish (Danio rerio). Aquat Toxicol. 2015;159:119-26.

71. Xu H, Dong X, Zhang Z, Yang M, Wu X, Liu H, Lao Q, Li C. Assessment of immunotoxicity of dibutyl phthalate using live zebrafish embryos. Fish Shellfish Immunol. 2015;45:286-92.

72. Bolognesi C. Genotoxicity of pesticides: a review of human biomonitoring studies. Mutat Res. 2003:543:251-72.

73. Cambier S, Gonzalez P, Durrieu G, Bourdineaud JP. Cadmium-induced genotoxicity in zebrafish at environmentally relevant doses. Ecotoxicol Environ Saf. 2010;73:312-9.

74. Dedeh A, Ciutat A, Treguer-Delapierre M, Bourdineaud JP. Impact of gold nanoparticles on zebrafish exposed to a spiked sediment. Nanotoxicology. 2015;9:71-80.

75. Geffroy B, Ladhar C, Cambier S, Treguer-Delapierre M, Brethes D, Bourdineaud JP. Impact of dietary gold nanoparticles in zebrafish at very low contamination pressure: the role of size, concentration and exposure time. Nanotoxicology. 2012;6:144-60.

76. Segura-Aguilar J, Kostrzewa RM. Neurotoxins and neurotoxicity mechanisms. An overview. Neurotox Res. 2006;10:263-87.

77. Win-Shwe TT, Fujimaki H. Nanoparticles and neurotoxicity. Int J Mol Sci. 2011;12:6267-80.

78. Chakraborty C, Sarkar B, Hsu CH, Wen ZH, Lin CS, Shieh PC. Future prospects of nanoparticles on brain targeted drug delivery. J Neurooncol. 2009;93:285-6.

79. Morimoto Y, Kobayashi N, Shinohara N, Myojo T, Tanaka I, Nakanishi J. Hazard assessments of manufactured nanomaterials. J Occup Health. 2010;52:325-34

80. Daroczi B, Kari G, McAleer MF, Wolf JC, Rodeck U, Dicker AP. In vivo radioprotection by the fullerene nanoparticle DF-1 as assessed in a zebrafish model. Clin Cancer Res. 2006;12:7086-91.

81. Sheng L, Wang L, Su M, Zhao X, Hu R, Yu X, Hong J, Liu D, Xu B, Zhu $Y$, et al. Mechanism of $\mathrm{TiO}_{2}$ nanoparticle-induced neurotoxicity in zebrafish (Danio rerio). Environ Toxicol. 2014:31:163-75.

82. Braydich-Stolle L, Hussain S, Schlager JJ, Hofmann MC. In vitro cytotoxicity of nanoparticles in mammalian germline stem cells. Toxicol Sci. 2005:88:412-9.

83. Wang J, Zhu X, Zhang X, Zhao Z, Liu H, George R, Wilson-Rawls J, Chang $Y$, Chen Y. Disruption of zebrafish (Danio rerio) reproduction upon chronic exposure to TiO(2) nanoparticles. Chemosphere. 2011;83:461-7.

84. Duan J, Yu Y, Shi H, Tian L, Guo C, Huang P, Zhou X, Peng S, Sun Z. Toxic effects of silica nanoparticles on zebrafish embryos and larvae. PLoS ONE. 2013;8:e74606

85. Zhu X, Zhu L, Duan Z, Qi R, Li Y, Lang Y. Comparative toxicity of several metal oxide nanoparticle aqueous suspensions to Zebrafish (Danio rerio) early developmental stage. J Environ Sci Health Part A. 2008;43:278-84.

86. Hua J, Vijver MG, Richardson MK, Ahmad F, Peijnenburg WJ. Particlespecific toxic effects of differently shaped zinc oxide nanoparticles to zebrafish embryos (Danio rerio). Environ Toxicol Chem. 2014;33:2859-68.

87. Chakraborty C, Pal S, Doss G, Wen Z-H, Lin C-S. Nanoparticles as 'smart' pharmaceutical delivery. Front Biosci. 2012;18:1030-50.

88. Pryor JB, Harper BJ, Harper SL. Comparative toxicological assessment of PAMAM and thiophosphoryl dendrimers using embryonic zebrafish. Int J Nanomedicine. 2014;9:1947-56.

89. Mathias JR, Saxena MT, Mumm JS. Advances in zebrafish chemical screening technologies. Future Med Chem. 2012;4:1811-22.

90. Hermsen SA, van den Brandhof EJ, van der Ven LT, Piersma AH. Relative embryotoxicity of two classes of chemicals in a modified zebrafish embryotoxicity test and comparison with their in vivo potencies. Toxicol In Vitro. 2011;25:745-53.

91. Padilla S, Corum D, Padnos B, Hunter DL, Beam A, Houck KA, Sipes N, Kleinstreuer N, Knudsen T, Dix DJ, Reif DM. Zebrafish developmental screening of the toxcast phase I chemical library. Reprod Toxicol. 2012;33:174-87.

92. Hussainzada N, Lewis JA, Baer CE, Ippolito DL, Jackson DA, Stallings JDI. Whole adult organism transcriptional profiling of acute metal exposures in male zebrafish. BMC Pharmacol Toxicol. 2014;15:15.
93. Weiss C, Diabate $S$. A special issue on nanotoxicology. Arch Toxicol. 2011:85:705-6.

94. Donaldson K, Stone V, Tran CL, Kreyling W, Borm PJ. Nanotoxicology. Occup Environ Med. 2004;61:727-8.

95. Jang GH, Hwang MP, Kim SY, Jang HS, Lee KH. A systematic in-vivo toxicity evaluation of nanophosphor particles via zebrafish models. Biomaterials. 2014;35:440-9.

96. Czupryna J, Tsourkas A. Suicide gene delivery by calcium phosphate nanoparticles: a novel method of targeted therapy for gastric cancer. Cancer Biol Ther. 2006;5:1691-2.

97. Yoon KY, Hoon Byeon J, Park JH, Hwang J. Susceptibility constants of Escherichia coli and Bacillus subtilis to silver and copper nanoparticles. Sci Total Environ. 2007;373:572-5.

98. Jin S, Ye K. Nanoparticle-mediated drug delivery and gene therapy. Biotechnol Prog. 2007:23:32-41.

99. Prow T, Grebe R, Merges C, Smith J, McLeod S, Leary J, Lutty G. Nanoparticle tethered biosensors for autoregulated gene therapy in hyperoxic endothelium. Nanomed Nanotechnol Biol Med. 2006;2:276.

100. Perugini P, Simeoni S, Scalia S, Genta I, Modena T, Conti B, Pavanetto F. Effect of nanoparticle encapsulation on the photostability of the sunscreen agent, 2-ethylhexyl-p-methoxycinnamate. Int J Pharm. 2002:246:37-45

101. Lee KJ, Browning LM, Nallathamby PD, Desai T, Cherukuri PK, Xu XH. In vivo quantitative study of sized-dependent transport and toxicity of single silver nanoparticles using zebrafish embryos. Chem Res Toxicol. 2012;25:1029-46.

102. Bar-llan O, Albrecht RM, Fako VE, Furgeson DY. Toxicity assessments of multisized gold and silver nanoparticles in zebrafish embryos. Small. 2009;5:1897-910.

103. George S, Lin S, Ji Z, Thomas CR, Li L, Mecklenburg M, Meng H, Wang X, Zhang $H$, Xia T, et al. Surface defects on plate-shaped silver nanoparticles contribute to its hazard potential in a fish gill cell line and zebrafish embryos. ACS Nano. 2012;6:3745-59.

104. George S, Gardner H, Seng EK, Chang H, Wang C. Yu Fang CH, Richards M, Valiyaveettil S, Chan WK: Differential effect of solar light in increasing the toxicity of silver and titanium dioxide nanoparticles to a fish cell line and zebrafish embryos. Environ Sci Technol. 2014;48:6374-82.

105. Lee KJ, Browning LM, Nallathamby PD, Xu XH. Study of charge-dependent transport and toxicity of peptide-functionalized silver nanoparticles using zebrafish embryos and single nanoparticle plasmonic spectroscopy. Chem Res Toxicol. 2013;26:904-17.

106. Choi JE, Kim S, Ahn JH, Youn P, Kang JS, Park K, Yi J, Ryu DY. Induction of oxidative stress and apoptosis by silver nanoparticles in the liver of adult zebrafish. Aquat Toxicol. 2010;100:151-9.

107. Xin Q, Rotchell JM, Cheng J, Yi J, Zhang Q. Silver nanoparticles affect the neural development of zebrafish embryos. J Appl Toxicol. 2015;35:1481-92.

108. Devi GP, Ahmed KB, Varsha MK, Shrijha BS, Lal KK, Anbazhagan V, Thiagarajan R. Sulfidation of silver nanoparticle reduces its toxicity in zebrafish. Aquat Toxicol. 2015:158:149-56.

109. Sharma G, Sharma AR, Kurian M, Bhavesh R, Nam JS, Lee SS. Green synthesis of silver nanoparticle using Myristica fragrans (nutmeg) seed extract and its biological activity. Digest J Nanomat Biostruc. 2014:9:325-32.

110. Singhal G, Bhavesh R, Sharma AR, Singh RP. Ecofriendly biosynthesis of gold nanoparticles using medicianally important Ocimum basilicum leaf extract. Adv Sci Eng Med. 2012;4:62-6.

111. Huang X, El-Sayed IH, Qian W, El-Sayed MA. Cancer cell imaging and photothermal therapy in the near-infrared region by using gold nanorods. J Am Chem Soc. 2006;128:2115-20.

112. Lim ZZ, Li JE, Ng CT, Yung LY, Bay BH. Gold nanoparticles in cancer therapy. Acta Pharmacol Sin. 2011;32:983-90.

113. Wang G, Stender AS, Sun W, Fang N. Optical imaging of non-fluorescent nanoparticle probes in live cells. Analyst. 2010;135:215-21.

114. Klein S, Petersen S, Taylor U, Rath D, Barcikowski S. Quantitative visualization of colloidal and intracellular gold nanoparticles by confocal microscopy. J Biomed Opt. 2010;15:036015.

115. Dykman LA, Khlebtsov NG. Gold nanoparticles in biology and medicine: recent advances and prospects. Acta Naturae. 2011;3:34-55.

116. Goodman CM, McCusker CD, Yilmaz T, Rotello VM. Toxicity of gold nanoparticles functionalized with cationic and anionic side chains. Bioconjug Chem. 2004;15:897-900. 
117. Gerber A, Bundschuh M, Klingelhofer D, Groneberg DA. Gold nanoparticles: recent aspects for human toxicology. J Occup Med Toxicol. 2013;8:32.

118. Kim KT, Zaikova T, Hutchison JE, Tanguay RL. Gold nanoparticles disrupt zebrafish eye development and pigmentation. Toxicol Sci. 2013;133:275-88.

119. Harper SL, Carriere JL, Miller JM, Hutchison JE, Maddux BL, Tanguay RL. Systematic evaluation of nanomaterial toxicity: utility of standardized materials and rapid assays. ACS Nano. 2011;5:4688-97.

120. He H, Pham-Huy LA, Dramou P, Xiao D, Zuo P, Pham-Huy C. Carbon nanotubes: applications in pharmacy and medicine. Biomed Res Int 2013;2013:578290

121. Liu Z, Tabakman S, Welsher K, Dai H. Carbon nanotubes in biology and medicine: in vitro and in vivo detection imaging and drug delivery. Nano Res. 2009;2:85-120.

122. Madani SY, Mandel A, Seifalian AM. A concise review of carbon nanotube's toxicology. Nano Rev. 2013;4

123. Ali-Boucetta H, Al-Jamal KT, Kostarelos K. Cytotoxic assessment of carbon nanotube interaction with cell cultures. Methods Mol Biol. 2011;726:299-312.

124. Luanpitpong S, Wang L, Rojanasakul Y. The effects of carbon nanotubes on lung and dermal cellular behaviors. Nanomedicine (Lond). 2014:9:895-912

125. Maes HM, Stibany F, Giefers S, Daniels B, Deutschmann B, Baumgartner W, Schaffer A. Accumulation and distribution of multiwalled carbon nanotubes in zebrafish (Danio rerio). Environ Sci Technol. 2014:48:12256-64.

126. Li J, Ying GG, Jones KC, Martin FL. Real-world carbon nanoparticle exposures induce brain and gonadal alterations in zebrafish (Danio rerio) as determined by biospectroscopy techniques. Analyst. 2015;140:2687-95.

127. Cheng J, Cheng SH. Influence of carbon nanotube length on toxicity to zebrafish embryos. Int J Nanomedicine. 2012;7:3731-9.

128. FilhoJde S, Matsubara EY, Franchi LP, Martins IP, Rivera LM, Rosolen JM, Grisolia CK. Evaluation of carbon nanotubes network toxicity in zebrafish (Danio rerio) model. Environ Res. 2014;134:9-16.

129. Fernández-García M, Rodriguez JA. Metal oxide nanoparticles. Encyclopedia Inorganic Bioinorganic Chem. 2007.

130. Franke ME, Koplin TJ, Simon U. Metal and metal oxide nanoparticles in chemiresistors: does the nanoscale matter? Small. 2006;2:36-50.

131. Jeng HA, Swanson J. Toxicity of metal oxide nanoparticles in mammalian cells. J Environ Sci Health A Tox Hazard Subst Environ Eng. 2006:41:2699-711.

132. Zhu X, Zhu L, Duan Z, Qi R, Li Y, Lang Y. Comparative toxicity of several metal oxide nanoparticle aqueous suspensions to Zebrafish (Danio rerio) early developmental stage. J Environ Sci Health A Tox Hazard Subst Environ Eng. 2008:43:278-84.
133. Palaniappan PR, Pramod KS. The effect of titanium dioxide on the biochemical constituents of the brain of Zebrafish (Danio rerio): an FT-IR study. Spectrochim Acta A Mol Biomol Spectrosc. 2011;79:206-12.

134. Palaniappan PLRM, Pramod KS. Raman spectroscopic investigation on the microenvironment of the liver tissues of Zebrafish (Danio rerio) due to titanium dioxide exposure. Vib Spectrosc. 2011;56:46-153.

135. Chen J, Dong $X$, Xin $Y$, Zhao M. Effects of titanium dioxide nano-particles on growth and some histological parameters of zebrafish (Danio rerio) after a long-term exposure. Aquat Toxicol. 2011;101:493-9.

136. Lin S, Zhao Y, Xia T, Meng H, Ji Z, Liu R, George S, Xiong S, Wang $X$, Zhang $H$, et al. High content screening in zebrafish speeds up hazard ranking of transition metal oxide nanoparticles. ACS Nano. 2011;5:7284-95.

137. Bar-Ilan O, Louis KM, Yang SP, Pedersen JA, Hamers RJ, Peterson RE Heideman W. Titanium dioxide nanoparticles produce phototoxicity in the developing zebrafish. Nanotoxicology. 2012;6:670-9.

138. Kanungo J, Cuevas E, Ali SF, Paule MG. Zebrafish model in drug safety assessment. Curr Pharm Des. 2014:20:5416-29.

139. Spitsbergen JM, Kent ML. The state of the art of the zebrafish model for toxicology and toxicologic pathology research-advantages and current limitations. Toxicol Pathol. 2003;31(Suppl):62-87.

140. Chang J, Ichihara G, Shimada Y, Tada-Oikawa S, Kuroyanagi J, Zhang B, Suzuki Y, Sehsah R, Kato M, Tanaka T, Ichihara S. Copper oxide nanoparticles reduce vasculogenesis in transgenic zebrafish through down-regulation of vascular endothelial growth factor expression and induction of apoptosis. J Nanosci Nanotechnol. 2015;15:2140-7.

141. Jovanovic B, Ji T, Palic D. Gene expression of zebrafish embryos exposed to titanium dioxide nanoparticles and hydroxylated fullerenes. Ecotoxicol Environ Saf. 2011;74:1518-25.

142. Burns CG, Milan DJ, Grande EJ, Rottbauer W, MacRae CA, Fishman MC. High-throughput assay for small molecules that modulate zebrafish embryonic heart rate. Nat Chem Biol. 2005;1:263-4.

143. Patra CR, Kim JH, Pramanik K, d'Uscio LV, Patra S, Pal K, Ramchandran R, Strano MS, Mukhopadhyay D. Reactive oxygen species driven angiogenesis by inorganic nanorods. Nano Lett. 2011;11:4932-8.

144. Zhang W, Lin K, Sun X, Dong Q, Huang C, Wang H, Guo M, Cui X. Toxicological effect of MPA-CdSe QDs exposure on zebrafish embryo and larvae. Chemosphere. 2012;89:52-9.

145. Sharif F, Porta F, Meijer AH, Kros A, Richardson MK. Mesoporous silica nanoparticles as a compound delivery system in zebrafish embryos. Int J Nanomed. 2012;7:1875-90.

\section{Submit your next manuscript to BioMed Central and we will help you at every step:}

- We accept pre-submission inquiries

- Our selector tool helps you to find the most relevant journal

- We provide round the clock customer support

- Convenient online submission

- Thorough peer review

- Inclusion in PubMed and all major indexing services

- Maximum visibility for your research

Submit your manuscript at www.biomedcentral com/submit 